\title{
The efficacy and safety of Osimertinib in advanced non-small cell lung cancer patients with Thr790Met resistance mutations: a systematic review and meta-analysis
}

\author{
Saijin Cui, Yaling Zhang, Lu Liu, Yan Li, Rongmiao Zhou, Xi Huang, Shiru Cao, Xiangran Huo, \\ Na Wang \\ Cancer Institute, The Fourth Hospital of Hebei Medical University, Shijiazhuang, China \\ Contributions: (I) Conception and design: N Wang, Y Li, S Cui; (II) Administrative support: N Wang, Y Li, R Zhou; (III) Provision of study materials \\ or patients: None; (IV) Collection and assembly of data: Y Zhang, L Liu, X Huang; (V) Data analysis and interpretation: S Cui, S Cao, X Huo; (VI) \\ Manuscript writing: All authors; (VII) Final approval of manuscript: All authors. \\ Correspondence to: Na Wang. Cancer Institute, The Fourth Hospital of Hebei Medical University, Shijiazhuang 050011, China. \\ Email: hbykdxwn@163.com.
}

\begin{abstract}
Background: Osimertinib, a third-generation epidermal growth factor receptor-tyrosine kinase inhibitor (EGFR-TKI), has been approved by the U.S. Food and Drug Administration in treating T790M mutationpositive advanced non-small cell lung cancer (NSCLC). A systematic review and meta-analysis was conducted to assess the efficacy and safety of osimertinib in treating advanced NSCLC patients with acquired T790M mutation.
\end{abstract}

Methods: PubMed, EMBASE, Cochrane Library and Web of Science were searched to obtain the eligible studies following the "population, interventions, comparisons, outcomes, study design" (PICOS) criteria. The pooled analysis of objective response rate (ORR), disease controlled rate (DCR), progressionfree survival (PFS), overall survival (OS) and adverse events (AEs) were performed using STATA12.0 and RevMan5.0.

Results: A total of 1,050 patients were included in the meta-analysis. The combined osimertinib ORR was 0.64 (95\% CI, 0.60-0.69), the ORR of central nervous system (CNS) was 0.54 (95\% CI, 0.37-0.71), DCR was 0.89 (95\% CI, 0.86-0.92), PFS at six months (PFS-6m) rate was 0.69 (95\% CI, 0.58-0.79), PFS at one year (PFS-1y) rate was 0.33 (95\% CI, 0.20-0.46), OS at one year (OS-1y) rate was 0.69 (95\% CI, 0.55-0.84). The pooled incidence rate of the AEs of grade $\geq$ III was 0.25 (95\% CI, 0.09-0.40). The results from Begg's and Egger's tests presented no publication bias in the included studies.

Conclusions: Osimertinib demonstrated a superior therapeutic benefit with high efficacy and low toxicity for T790M-positive advanced NSCLC patients who were treated with early-generation EGFR-TKIs. Meanwhile, osimertinib showed promising for the treatment of advanced patients with CNS metastases.

Keywords: Osimertinib; advanced non-small cell lung cancer (NSCLC); T790M mutation; central nervous system (CNS); meta-analysis

Submitted Jul 03, 2020. Accepted for publication Oct 23, 2020.

doi: 10.21037/apm-20-1357

View this article at: http://dx.doi.org/10.21037/apm-20-1357

\section{Introduction}

Lung cancer ranks first in cancer incidence and mortality worldwide (1). Non-small cell lung cancer (NSCLC) accounts for approximately $85 \%$ of all lung cancer, and most
NSCLC patients are diagnosed at an advanced stage $(2,3)$. The activation of epidermal growth factor receptor (EGFR) mutations, one of most common genetic event, has been identified as oncogenic drivers for NSCLC (4). However, 
conventional chemotherapy showed poor treatment efficacy and prognosis in treating EGFR mutation-positive advanced NSCLC patients. Recently, specific targeted therapies using EGFR- tyrosine kinase inhibitors (TKIs), which block the growth of cancer cells through interfering with EGFR gene, have demonstrated significant clinical benefit and become first-line treatment in advanced NSCLC patients with an EGFR driver mutation (5-9). Despite impressive tumor responses with first-line EGFR-TKIs, acquired drug resistance occurs inevitably in most EGFR mutant advanced NSCLC patients (10).

Thr790Met (T790M) mutation in exon 20, detected in more than half of the patients who develop resistance to EGFR-TKIs, is recognized as the dominant acquired resistance mechanisms (11). The resistance mechanism of EGFR-T790M mutation is that the presence of T790M variant increases the affinity of adenosinetriphosphate (ATP) for the EGFR active site, and reduces the binding ability of the EGFR-TKIs relatively, resulting in reduced inhibition of EGFR-TKIs-mediated downstream signaling pathway (12). T790M mutation were detected in EGFRmutant advanced NSCLC patients after drug resistance in first-generation EGFR-TKIs within 9-14 months (13). The second-generation EGFR-TKIs were developed to overcome EGFR-T790M mutation-related resistance by irreversibly binding to the cysteine residue of $\operatorname{EGFR}(14,15)$. However, these TKIs were non-selective between EGFRT790M mutant and wild-type EGFR. They also had potent against wild-type EGFR and lead to severe toxicity that limited clinical doses required to inhibit T790M mutation effectively. Moreover, the second-generation EGFR-TKIs did not show improvement in treating patients who had received treatment with first-generation TKIs $(16,17)$. Thus, the clinical utility of second-generation EGFR-TKIs remains questionable in advanced NSCLC patients with T790M mutation following disease progression on the firstgeneration TKIs.

Osimertinib is an oral, central nervous system (CNS) active, irreversible third-generation EGFR-TKI that has potent against both the EGFR mutations and the EGFRT790M resistance mutation selectivity, with a minimal inhibition of the wild-type EGFR (18). Osimertinib showed stronger potency against T790M mutation at cellular level compared with early generation TKIs. Studies in vivo using xenograft and transgenic tumor models suggested that osimertinib produced profound and sustained regression in EGFR-T790M mutant tumors (19). In addition, osimertinib demonstrated markedly penetration of blood-brain barrier (BBB) and induced durable shrinkage in mouse and nonhuman primate brain models (20). Currently, several clinical trials have been reported, but there was a discrepancy in the outcomes of osimertinib for the treatment of EGFR-T790M mutationpositive advanced NSCLC (21-26). In the present study, we conducted a systematic review and meta-analysis aiming to provide comprehensive clinical evidence for evaluating the efficacy and safety of osimertinib in treating these patients. We present the following article in accordance with the PRISMA reporting checklist (available at http://dx.doi. org/10.21037/apm-20-1357).

\section{Methods}

\section{Search strategy}

The Preferred Reporting Items for Systematic Reviews and Meta-Analyses (PRISMA) guidelines were followed for our systematic review and meta-analysis. PubMed, EMBASE, the Cochrane Library, and Web of Science were searched for clinical trials of osimertinib in treating EGFR-T790M advanced NSCLC published before July 29, 2019. Abstracts and presentations from all major conference proceedings were also reviewed, including the American Society of Clinical Oncology (ASCO) and the European Society for Medical Oncology (ESMO), from January 1, 2010, to July 29, 2019. The search terms were "Osimertinib", "Tagrisso", "AZD9291", "non-small cell lung cancer", "NSCLC", "EGFR", "T790M". The references of enrolled studies were reviewed in the final selection to ensure that no trails were missed.

\section{Study selection}

Studies meeting the following PICOS criteria were included: (I) patients: advanced NSCLC patients with EGFR T790M-positive; (II) intervention: treatment with Osimertinib; (III) comparison: treatment with at least one of the previous EGFR-TKIs (Gefitinib/Erlotinib/Afatinib/ Dacomitinib); (IV) outcome: at least one of the following data: objective response rate (ORR), disease controlled rate (DCR), progression-free survival (PFS), overall survival (OS) and adverse events (AEs) were reported; (V) study design: the randomized controlled trial (RCT) or prospective phase I, II, and III trial. Exclusion criteria were as follows: (I) reviews, letters, editorials, meeting, repeated data or overlapping studies; (II) studies on cellular or animal; (III) 
providing insufficient data for estimating the results.

\section{Data extraction and quality assessment}

The data extraction was carried out independently by two reviewers (Lu Liu, Yaling Zhang), in accordance with PRISMA. Any discrepancies were resolved by discussion and consensus. The following baseline information was collected from the eligible studies: name of the first author, year of publication, phase of the trial, sample size, previous EGFR-TKIs treatment, outcomes of treatment effect (ORR, DCR, median PFS, OS-1y rate) and toxicity.

To assess the methodological quality of the included articles, the Cochrane Collaboration's "risk of bias" (ROB) tool and Newcastle-Ottawa Scale (NOS) were used, which was also performed independently by two reviewers. The Cochrane Collaboration's ROB tool was used to evaluate the RCTs. The overall ROB of a study were defined as "low" if the "low risk" items were more than four, "medium" if the "low risk" items were two or three, and "high" if the "low risk" items were fewer than two or "high risk" items were more than one. The NOS was used to estimate the single-arm trials (27). The NOS quality evaluation consists of three aspects (Appendix 1): selection (0-4 points), comparability ( $0-2$ points), and outcome ( $0-3$ points). The quality of each study was defined as "poor" when the total score was less than 4, "fair" when the score ranged from 4 to 6 ,and "good" when the score ranged from 7 to 9 (28).

\section{Statistical analysis}

Pooled data management and analysis were performed by using STATA12.0 (provided by Stata Corp) and Review Manager 5.0 software (provided by Cochrane Collaboration). The heterogeneity among the retrieved studies was evaluated by using Higgins inconsistency index $\left(\mathrm{I}^{2}\right)$ test. The fixed-effects models was used if $\mathrm{I}^{2}<50 \%$ $(\mathrm{P}>0.1)$, otherwise, the random-effect model was applied. Statistical analysis of ORR, DCR, PFS-6m rate, PFS$1 \mathrm{y}$ rate, OS-1y rate and AEs of all grades or of grade $\geq$ III were combined with the corresponding $95 \%$ CI. The pooled ORR of patients with CNS metastases was calculated as well. When PFS or OS were not reported directly in studies, the data were extracted from the survival curves as reported. A two-sided $\mathrm{P}$ value of $<0.05$ was considered statistically significant. Publication bias of the included studies was evaluated by using Begg's and
Egger's test.

\section{Results}

\section{Search results}

Based on our search strategy, 515 potentially relevant studies were initially identified, of which 118 articles were excluded for duplication, 378 articles were excluded after titles and abstracts screening, 13 articles were excluded after full-text reviewing. As a result, the remaining 6 articles were enrolled for our meta-analysis (21-26). The selection process was presented in Figure 1.

\section{Characteristics of the included studies}

A total of six eligible clinical studies including 1010 EGFRT790M advanced NSCLC patients were enrolled in this analysis. The number of patients in each trails ranged 74 to 279 , and the included studies were published from 2016 to 2018. All eligible trials were carried out to estimate the efficacy and safety of osimertinib including three RCTs (Mok 2017, Nie 2017 and Wu 2018) (21-23) and three single-arm trials (Yang 2017, Goss 2016, and Zhou 2017) (24-26). Additionally, three among all studies evaluated the CNS impact of osimertinib treatment in the patients (23-25). Detailed baseline characteristics of the enrolled studies were summarized in Table 1. There was no "high risk" or "poor" for quality assessment of our included studies (the detailed quality assessment of included studies was reported in Table S1 and Figure S1).

\section{Efficacy}

Six studies provided the data on ORR and DCR of osimertinib in the treatment of EGFR T790M-positive advanced NSCLC. Those tests for heterogeneity were significant, and the random-effects model was applied. The pooled ORR and DCR were 0.64 (95\% CI, 0.60-0.69; $\mathrm{I}^{2}=51.2 \%, \mathrm{P}=0.084$; Figure $\left.2 A\right)$ and 0.89 (95\% CI, 0.86$0.92 ; \mathrm{I}^{2}=51.7 \%, \mathrm{P}=0.082 ;$ Figure $\left.2 B\right)$, respectively. Besides, the pooled CNS ORR was 0.54 (95\% CI, 0.37-0.71; $\mathrm{I}^{2}=86.5 \%, \mathrm{P}=0.001$; Figure $2 C$ ). The data on PFS and OS were available from four studies. The combined PFS$6 \mathrm{~m}$ rate, PFS-1y rate and OS-1y rate were 0.69 (95\% CI, 0.58-0.79; $\mathrm{I}^{2}=86.2 \%, \mathrm{P}<0.001$; Figure $3 A$ ), 0.33 (95\% CI, $0.20-0.46 ; \mathrm{I}^{2}=93.1 \%, \mathrm{P}<0.001$; Figure $\left.3 B\right)$, and $0.69(95 \%$ CI, 0.55-0.84; $\mathrm{I}^{2}=92.2 \%, \mathrm{P}<0.001$; Figure 3 C), respectively. 


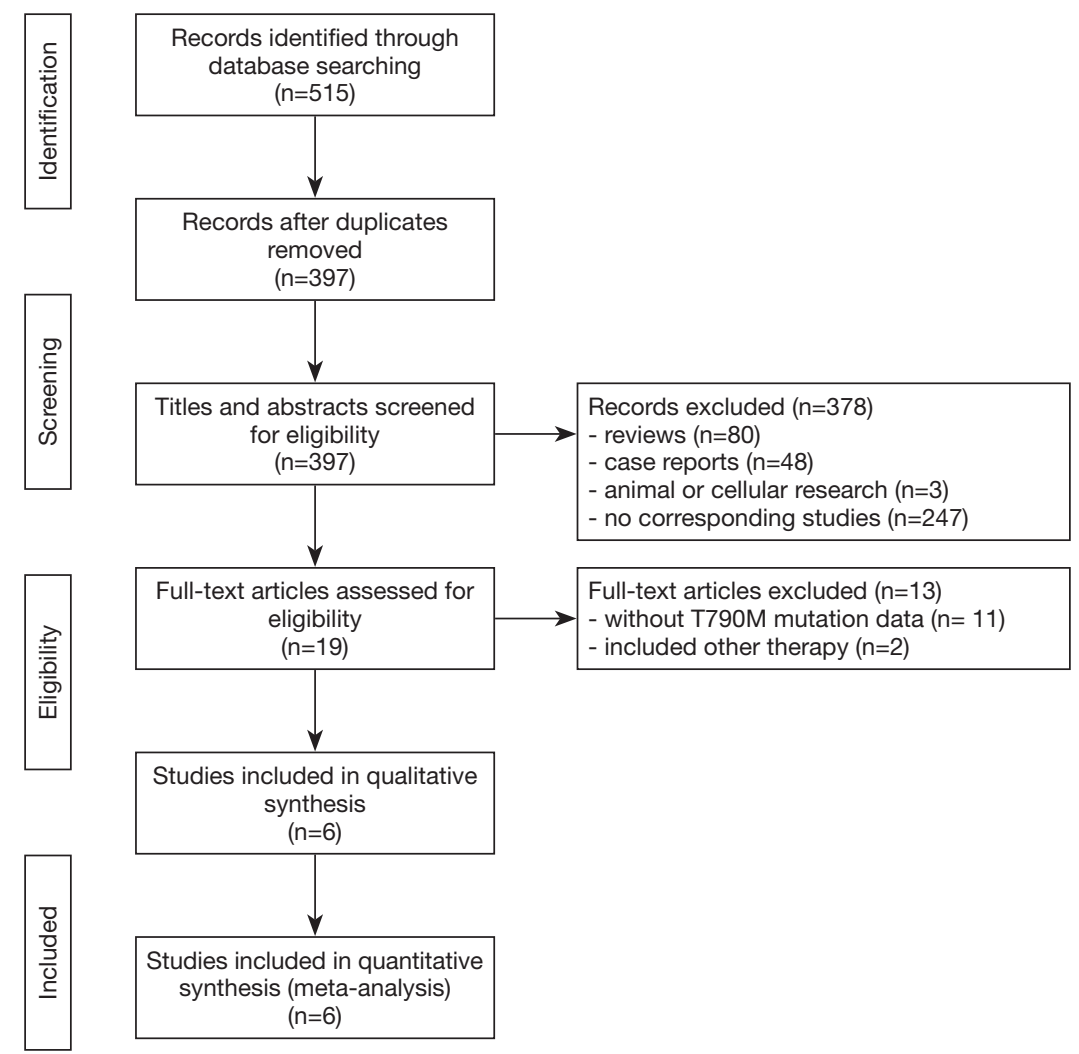

Figure 1 Flow chart of the literature search.

Table 1 Baseline characteristics of included studies in the meta-analysis

\begin{tabular}{|c|c|c|c|c|c|c|c|c|}
\hline Study (year) & Phase & $\begin{array}{l}\text { Sample } \\
\text { size, } n\end{array}$ & $\begin{array}{c}\text { Previous } \\
\text { EGFR-TKI (\%) }\end{array}$ & ORR (\%) & DCR (\%) & $\begin{array}{c}\text { Median PFS (m) } \\
(95 \% \mathrm{Cl})\end{array}$ & $\begin{array}{c}\text { OS-1y } \\
\text { rate (\%) }\end{array}$ & Quality score \\
\hline Mok (2016) & III & 279 & $\begin{array}{c}\text { Gefitinib/erlotinib/ } \\
\text { afatinib (59\%/34\%/7\%) }\end{array}$ & 198/279 (70.97) & $260 / 279(91.3)$ & $10.1(8.3-12.3)$ & NR & $\begin{array}{l}\text { Cochrane ROB } \\
\text { Tool: Low risk }\end{array}$ \\
\hline Nie (2018) & III & 74 & $\begin{array}{c}\text { Gefitinib/erlotinib } \\
(62.2 \% / 37.8 \%)\end{array}$ & $46 / 74(62.16)$ & $65 / 74(87.84)$ & 10.2 (NR) & $36 / 74(48.6)$ & $\begin{array}{l}\text { Cochrane ROB } \\
\text { Tool: Low risk }\end{array}$ \\
\hline Goss (2016) & II & 210 & $\begin{array}{c}\text { Gefitinib/erlotinib/ } \\
\text { afatinib/dacomitinib } \\
\text { (58\%/57\%/18\%/1\%) }\end{array}$ & $140 / 210(66.67)$ & $182 / 210(86.67)$ & $9.9(8.5-12.3)$ & $170 / 210(81)$ & 6 \\
\hline
\end{tabular}

EGFR-TKI, epidermal growth factor receptor tyrosine kinase inhibitors; PFS, progression-free survival; ORR, objective response rate; DCR, disease control rate; OS-1y rate, survival rate for 1 year; $m$, month; NR, not report; ROB, risk of bias. 


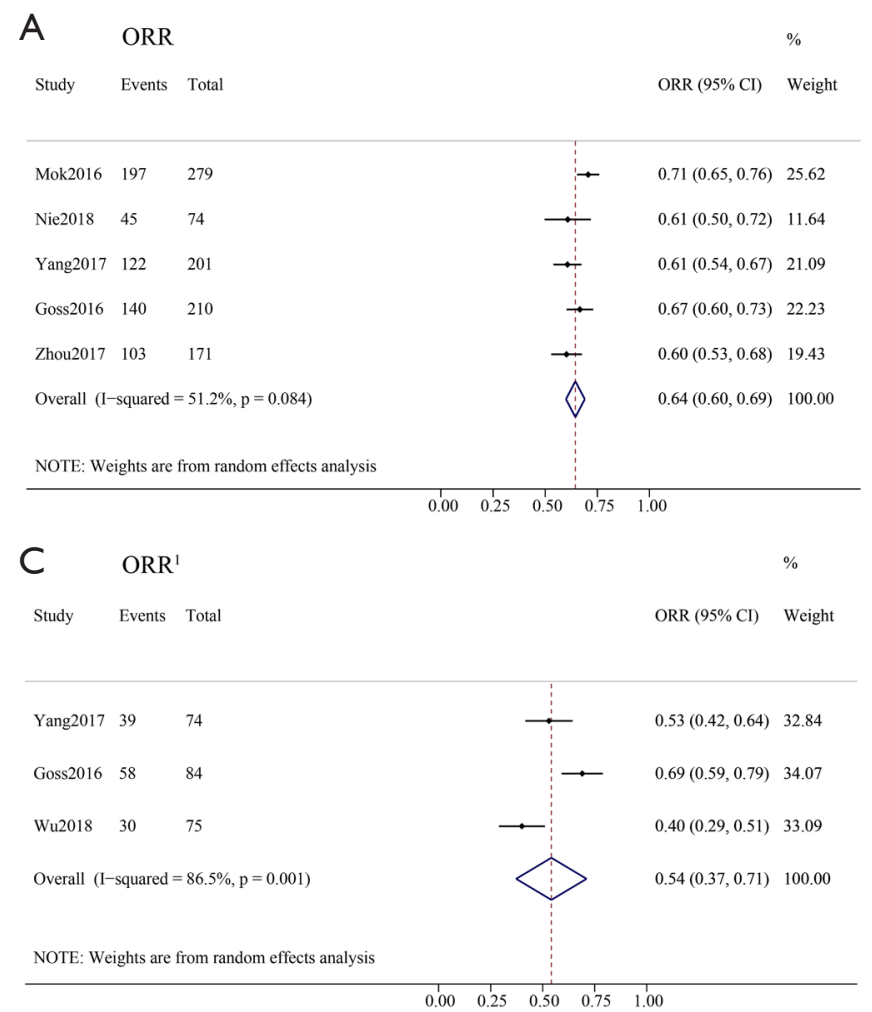

\begin{tabular}{|c|c|c|c|c|c|}
\hline B & DCR & & & & $\%$ \\
\hline Study & Events & Total & & DCR $(95 \% \mathrm{Cl})$ & Weight \\
\hline Mok2016 & 260 & 279 & - & $0.93(0.90,0.96)$ & 28.64 \\
\hline Nie2018 & 64 & 74 & $\rightarrow$ & $0.86(0.79,0.94)$ & 10.54 \\
\hline Yang2017 & 179 & 201 & - & $0.89(0.85,0.93)$ & 21.58 \\
\hline Goss2016 & 182 & 210 & $\rightarrow$ & $0.87(0.82,0.91)$ & 20.31 \\
\hline Zhou2017 & 150 & 171 & - & $0.88(0.83,0.93)$ & 18.95 \\
\hline Overall (I- & squared & $=51.7 \%, p=0.082)$ & 0 & $0.89(0.86,0.92)$ & 100.00 \\
\hline NOTE: We & ights are & from random effects analysis & & & \\
\hline
\end{tabular}

Figure 2 Forest plots of the efficacy of osimertinib treatment in EGFR-T790M mutant advanced NSCLC patients. (A) ORR, objective response rate; (B) DCR, disease control rate; (C) ORR ${ }^{1}$, objective response rate of osimertinib treatment in EGFR-T790M mutant advanced NSCLC patients with CNS metastases.

\section{Safety}

The analyses of AEs (all grades or of grade $\geq$ III) of osimertinib covered five trials. The most common AEs in all grade were rash or acne, diarrhea and dry skin, and results of the random-effects model showed that the combined incidence rate of those AEs were 0.34 (95\% CI, 0.26-0.42), 0.33 (95\% CI, 0.27-0.38) and 0.30 (95\% CI, 0.21-0.38), respectively (Figure 4). Regarding the AEs of grade $\geq \mathrm{III}$, the pooled incidence rate was 0.25 (95\% CI, 0.09-0.40, Figure $4 D$ ). The ranking pooled incidence rate of the AEs of grade $\geq$ III suggested that the highest pooled rate was anemia $(0.01,95 \%$ CI, 0.00-0.02), followed by dyspnea and asthenia (0.01, 95\% CI, 0.00-0.02). In addition, the combined rate of diarrhea (grade $\geq$ III) was 0.01 (95\% CI, $0.00-0.01$ ), and the pooled rate of rash or acne (grade $\geq$ III) was 0.01 (95\% CI, 0.00-0.01).

\section{Publication bias}

For each analysis of the efficacy and safety, Begg's and
Egger's tests were used to assess publication bias of the included studies. No evidence in the results of Begg's and Egger's tests suggested publication bias in those studies. The details of publication bias are shown in Table 2.

\section{Discussion}

The acquired resistance to first- and second-generation EGFR-TKIs has become a critical issue in the treatment of EGFR T790M-positive advanced NSCLC patients. Osimertinib, a third-generation EGFR-TKI, has been approved for the treatment in patients with EGFR T790Mpositive advanced NSCLC. Our studies conducted a metaanalysis based on all the evidence available on the efficacy and safety of osimertinib therapy for advanced NSCLC patients with T790M mutation after treatment with early generation EGFR-TKIs. The statistical analysis showed that the pooled ORR and DCR were $64 \%$ and $89 \%$, respectively, and the combined rate of PFS-6m, PFS$1 \mathrm{y}$ and OS-1y were $69 \%, 33 \%$, and $69 \%$, respectively, 


\begin{tabular}{|c|c|c|c|c|}
\hline \multicolumn{3}{|l|}{$\Delta$} & PFS-6m & $\%$ \\
\hline \multicolumn{3}{|l|}{ Study } & rate $(95 \% \mathrm{Cl})$ & Weight \\
\hline Mok2016 & $\rightarrow$ & & $0.58(0.50,0.66)$ & 25.42 \\
\hline $\mathrm{Nie} 2018$ & & $\rightarrow$ & $0.85(0.76,0.94)$ & 24.40 \\
\hline Yang2017 & & & $0.65(0.57,0.73)$ & 24.94 \\
\hline Goss2016 & & & $0.67(0.59,0.74)$ & 25.24 \\
\hline Overall $(\mathrm{I}-\mathrm{squared}=86.2 \%, \mathrm{p}=0.000)$ & & & $0.69(0.58,0.79)$ & 100.00 \\
\hline \multicolumn{5}{|l|}{ NOTE: Weights are from random effects analysis } \\
\hline \multicolumn{5}{|c|}{$0.00 \quad 0.25 \quad 0.50$} \\
\hline \multirow{2}{*}{\multicolumn{3}{|c|}{ Study }} & OS-1y & $\%$ \\
\hline & & & rate $(95 \% \mathrm{CI})$ & Weight \\
\hline $\mathrm{Nie} 2018$ & $\longrightarrow$ & & $0.49(0.37,0.60)$ & 30.08 \\
\hline Yang2017 & & $\rightarrow$ & $0.75(0.69,0.81)$ & 34.73 \\
\hline Goss2016 & & $\rightarrow$ & $0.81(0.76,0.86)$ & 35.19 \\
\hline Overall $(\mathrm{I}-\mathrm{squared}=92.2 \%, \mathrm{p}=0.000)$ & & & $0.69(0.55,0.84)$ & 100.00 \\
\hline NOTE: Weights are from random effects analysis & & & & \\
\hline
\end{tabular}

$\begin{array}{lllll}0.00 & 0.25 & 0.50 & 0.75 & 1.00\end{array}$

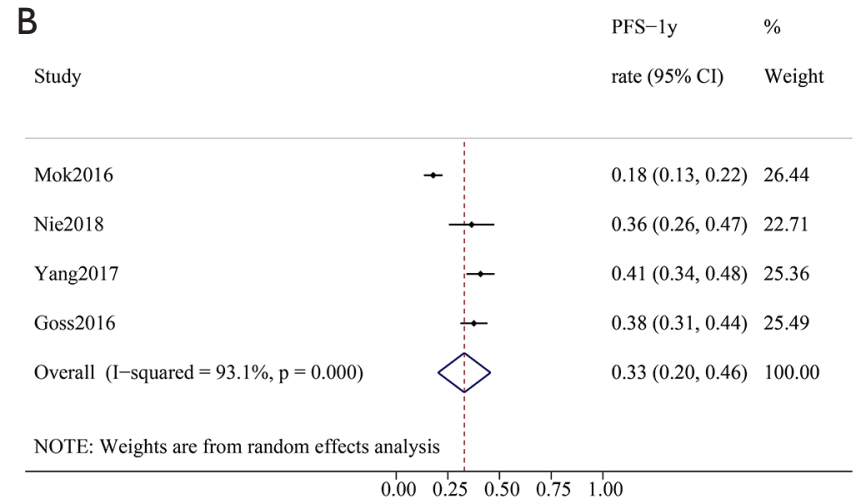

Figure 3 Forest plots of the efficacy of osimertinib treatment in advanced NSCLC patients with EGFR-T790M mutation. (A) PFS-6m rate, the proportion of patients in 6 months progression free survival; (B) PFS-1y rate, the proportion of patients in one year progression free survival; (C) OS-1y rate, the proportion of patients in one year overall survival.

which confirmed the satisfactory and persistent efficacy of osimertinib in treating EGFR-T790M mutant advanced NSCLC. In addition, the most common AEs rates were $34 \%$ for rash or acne, $33 \%$ for diarrhea and $30 \%$ for dry skin. The incidence of AEs of grade $\geq$ III was $25 \%$, with each grade 3 or higher AEs rate being controlled within $2 \%$, indicating a well tolerance. Moreover, the pooled CNS ORR of $54 \%$ demonstrated the encouraging effect of osimertinib on the patients with CNS metastases.

The treatment strategies for advanced NSCLC patients with acquired EGFR-T790M mutation after early generation TKI treatments are sparse. Platinum-based chemotherapy has become one of the common regimens for those patients. However, several previous studies showed dismal clinical effects (ORR: 26\%; PFS: 6.0 months) and significantly adverse events in patients received platinumbased chemotherapy (29-31). Moreover, it was useless to receive treatment with other first or second generation EGFR-TKIs for the patients who had acquired T790M mutation after first-line EGFR-TKIs therapy (16).
Osimertinib is now the only third-generation EGFRTKIs recommended for clinical use. Recently, an indirect comparison study showed that advanced NSCLC patients received osimertinib had better ORR (64.3\% vs. $33.3 \%)$ and PFS (10.9 vs. 5.3 months) compared to those received platinum-based doublet chemotherapy (32). Data from two published RCTs suggested that the clinical effects of osimertinib were superior to platinum-pemetrexed therapy and docetaxel-bevacizumab therapy in EGFR-T790M mutant advanced NSCLC.

In addition to efficacy, drug safety is another consideration for the drug assessment. Although dermatologic and gastro-intestinal toxicities remain the most common toxic effects, osimertinib has significantly lower incidence rate that previous generation EGFRTKIs (33). Meanwhile, osimertinib also reduce the risk of severe adverse events in patients when compared to other treatment regimens. Collectively, osimertinib demonstrated a superior optional strategy with the high efficacy and low toxicity for advanced NSCLC patients who acquired 


\begin{tabular}{llll} 
A & \multicolumn{2}{c}{$\%$} \\
Study & Proportion $(95 \% \mathrm{Cl})$ & Weight \\
\hline Mok2016 & $0.34(0.28,0.40)$ & 21.41 \\
Nie2018 & $0.36(0.25,0.47)$ & 16.62 \\
Yang2017 & $0.40(0.33,0.47)$ & 20.41 \\
Goss2016 & $0.41(0.34,0.48)$ & 20.51 \\
Zhou2017 & $0.20(0.14,0.26)$ & 21.06 \\
Overall (I-squared $=85.7 \%, p=0.000)$ & $0.34(0.26,0.42)$ & 100.00 \\
NOTE: Weights are from random effects analysis & & \\
\hline
\end{tabular}

$\begin{array}{lllll}0.00 & 0.25 & 0.50 & 0.75 & 1.00\end{array}$
B

\begin{tabular}{|c|c|c|c|}
\hline Diarrhea & & Proportion $(95 \% \mathrm{CI})$ & Weight \\
\hline Mok2016 & & $0.34(0.28,0.40)$ & 22.85 \\
\hline $\mathrm{Nie} 2018$ & & $0.26(0.16,0.36)$ & 14.85 \\
\hline Yang2017 & $\rightarrow$ & $0.43(0.36,0.50)$ & 20.32 \\
\hline Goss2016 & & $0.30(0.24,0.36)$ & 21.58 \\
\hline Zhou2017 & & $0.29(0.22,0.36)$ & 20.40 \\
\hline Overall $(\mathrm{I}-$ squared $=67.8 \%, \mathrm{p}=0.014)$ & & $0.33(0.27,0.38)$ & 100.00 \\
\hline NOTE: Weights are from random effects analysis & & & \\
\hline
\end{tabular}

D

\begin{tabular}{|c|c|c|c|}
\hline AEs of grade $\geq 3$ & & Proportion $(95 \% \mathrm{CI})$ & Weight \\
\hline Mok2016 & & $0.23(0.18,0.27)$ & 25.22 \\
\hline $\mathrm{Nie} 2018$ & & $0.04(0.00,0.09)$ & 25.32 \\
\hline Yang2017 & $\rightarrow$ & $0.38(0.32,0.45)$ & 24.68 \\
\hline Goss2016 & $\rightarrow$ & $0.34(0.28,0.41)$ & 24.78 \\
\hline Overall $(\mathrm{I}-\mathrm{squared}=96.9 \%, \mathrm{p}=0.000)$ & & $0.25(0.09,0.40)$ & 100.00 \\
\hline NOTE: Weights are from random effects analysis & & & \\
\hline
\end{tabular}

Figure 4 Forest plots of the safety of osimertinib treatment in EGFR-T790M mutant advanced NSCLC patients. (A) Rash or acne, the proportion of patients with rash or acne; (B) Diarrhea, the proportion of patients with diarrhea; (C) Dry skin, the proportion of patients with dry skin; (D) AEs of grade $\geq 3$, the proportion of patients with adverse events grade 3 or beyond.

Table 2 Begg's and Egger's tests

\begin{tabular}{lcc}
\hline Efficacy & Begg's test & Egger's test \\
\hline ORR & 0.462 & 0.193 \\
ORR $^{1}$ & 1.000 & 0.381 \\
DCR & 0.462 & 0.073 \\
PFS-6m rate & 0.308 & 0.060 \\
PFS-1y rate & 0.734 & 0.259 \\
OS-1y rate & 0.296 & 0.083 \\
$\geq$ grade3 AEs & 0.089 & 0.084 \\
\hline
\end{tabular}

ORR, objective response rate; $\mathrm{ORR}^{1}$, objective response rate of osimertinib treatment in EGFR-T790M mutant advanced NSCLC patients with CNS metastases; DCR, disease control rate; PFS$6 \mathrm{~m}$ rate, progression-free survival rate for six months; PFS-1y rate, progression-free survival rate for one year; OS-1y rate, survival rate for one year; AEs, adverse events.
T790M mutation following disease progression on the early generation EGFR-TKIs.

According to our data analysis on CNS metastases, osimertinib was impressively CNS active. Osimertinib had greater ability to penetrate the BBB than first- and secondgeneration TKIs in preclinical trials (20). A pooled data analysis of CNS metastases from two phase II clinical trials showed that osimertinib exhibited encouraging CNS ORR (54\%) and DCR (92\%) in advanced NSCLC patients who had EGFR T790M mutation and CNS metastases (34). In an AURA3 RCTs, compared with platinum-pemetrexed, osimertinib demonstrated a longer PFS (11.7 vs. 5.6 months), fewer CNS progression and lower incidence of new CNS lesions for the patients (23). These promising evidence provides a strong impetus for further research in the treatment of CNS metastases with osimertinib. 
Previous studies suggested a low incidence rate of T790M mutation in CNS due to the poor CNS penetration of early generation TKIs $(35,36)$. However, in a latest FLAURA study, osimertinib reduced the risk of CNS progression for EGFR mutant advanced NSCLC patients without T790M mutations (37). Therefore, osimertinib may be effective against both the T790M resistance mutation and EGFR sensitizing mutations for CNS metastases.

Recently, a global RCT showed that, first-line use of osimertinib had much better treatment efficacy than firstgeneration TKIs, especially for PFS (18.9 vs. 10.2 months), and did not find T790M resistance mutation was found in advanced EGFR mutant NSCLC patients (38). The highly clinical benefit of osimertinib as first-line treatment brought a new stage in advanced NSCLC therapy. Unfortunately, the follow-up clinical trials showed that acquired resistance has also inevitably emerged in the treatment of osimertinib and EGFR C797S mutation was the predominant resistance mechanism in both first- or later-line treatment with osimertinib (39). Interestingly, several case reports showed the sequential therapy of osimertinib followed by early generation TKIs prolonged the survival time (40-43). However, the patients still occurred progressive disease few months later. Therefore, the optimal strategy for the use of osimertinib in sequential therapy remains to be determined in further clinical trials. Given the evolving resistance mechanisms, there is an urgent need to develop new generation EGFR-TKIs.

Several limitations of our study should be considered. First, only three of the included articles were RCT, and all the others were single-arm trials, thus the significantly statistical conclusions were limited. Second, the overall statistical data were extracted from published articles without individual patient data. Third, the subgroup analysis of Exon 19 deletion and L858R in patients with EGFR-T790M mutation could not be performed due to the lack of sufficient data. Lastly, for patients who had CNS metastases, we only assessed the efficacy of osimertinib based on ORR because of the relatively deficiency details of CNS metastases in our meta-analysis. More clinical data such as PFS, OS and AEs from future studies are required to support our results.

\section{Conclusions}

The outcomes from our analysis demonstrate that osimertinib is a satisfactory optional strategy with the high efficacy and low toxicity in treating advanced NSCLC patients who acquired EGFR-T790M mutation after the treatment of early generation EGFR-TKIs. Osimertinib shows a promising efficacy for EGFR-T790M mutant advanced patients with CNS metastases. Moreover, further studies exploring sequential strategy of osimertinib and early generation TKIs are needed to provide appropriate clinical guidance.

\section{Acknowledgments}

Funding: This work was supported by the Natural Science Foundation of Hebei Province, Hebei, China. (Grant No. H2015206471).

\section{Footnote}

Reporting Checklist: The authors have completed the PRISMA reporting checklist. Available at http://dx.doi. org/10.21037/apm-20-1357

Conflicts of Interest: All authors have completed the ICMJE uniform disclosure form (available at http://dx.doi. org/10.21037/apm-20-1357). The authors have no conflicts of interest to declare.

Ethical Statement: The authors are accountable for all aspects of the work in ensuring that questions related to the accuracy or integrity of any part of the work are appropriately investigated and resolved.

Open Access Statement: This is an Open Access article distributed in accordance with the Creative Commons Attribution-NonCommercial-NoDerivs 4.0 International License (CC BY-NC-ND 4.0), which permits the noncommercial replication and distribution of the article with the strict proviso that no changes or edits are made and the original work is properly cited (including links to both the formal publication through the relevant DOI and the license). See: https://creativecommons.org/licenses/by-nc-nd/4.0/.

\section{References}

1. Bray F, Ferlay J, Soerjomataram I, et al. Global cancer statistics 2018: GLOBOCAN estimates of incidence and mortality worldwide for 36 cancers in 185 countries. CA Cancer J Clin 2018;68:394-424.

2. Ettinger DS, Borghaei H, Cheney RT, et al. Non-Small Cell Lung Cancer, Version 3.2012. J Natl Compr Canc 
Netw 2012;10:1236-71.

3. Wood DE, Kazerooni EA, Baum SL, et al. Lung Cancer Screening, Version 3.2018, NCCN Clinical Practice Guidelines in Oncology. J Natl Compr Canc Netw 2018;16:412-41.

4. Steuer CE, Khuri FR, Ramalingam SS. The next generation of epidermal growth factor receptor tyrosine kinase inhibitors in the treatment of lung cancer. Cancer 2015;121:E1-6.

5. Mitsudomi T, Morita S, Yatabe Y, et al. Gefitinib versus cisplatin plus docetaxel in patients with non-small-cell lung cancer harbouring mutations of the epidermal growth factor receptor (WJTOG3405): an open label, randomised phase 3 trial. Lancet Oncol 2010;11:121-8.

6. Rosell R, Carcereny E, Gervais R, et al. Erlotinib versus standard chemotherapy as first-line treatment for European patients with advanced EGFR mutation-positive non-small-cell lung cancer (EURTAC): a multicentre, open-label, randomised phase 3 trial. Lancet Oncol 2012;13:239-46.

7. Wu YL, Zhou C, Hu CP, et al. Afatinib versus cisplatin plus gemcitabine for first-line treatment of Asian patients with advanced non-small-cell lung cancer harbouring EGFR mutations (LUX-Lung 6): an open-label, randomised phase 3 trial. Lancet Oncol 2014;15:213-22.

8. Masters GA, Temin S, Azzoli CG, et al. Systemic Therapy for Stage IV Non-Small-Cell Lung Cancer: American Society of Clinical Oncology Clinical Practice Guideline Update. J Clin Oncol 2015;33:3488-515.

9. Ettinger DS, Aisner DL, Wood DE, et al. NCCN Guidelines Insights: Non-Small Cell Lung Cancer, Version 5.2018. J Natl Compr Canc Netw 2018;16:807-21.

10. Cortot AB, Janne PA. Molecular mechanisms of resistance in epidermal growth factor receptor-mutant lung adenocarcinomas. Eur Respir Rev 2014;23:356-66.

11. Yu HA, Arcila ME, Rekhtman N, et al. Analysis of Tumor Specimens at the Time of Acquired Resistance to EGFRTKI Therapy in 155 Patients with EGFR-Mutant Lung Cancers. Clin Cancer Res 2013;19:2240-47.

12. Yun CH, Mengwasser KE, Toms AV, et al. The T790M mutation in EGFR kinase causes drug resistance by increasing the affinity for ATP. Proc Natl Acad Sci U S A 2008; 105:2070-5.

13. Nagano T, Tachihara $M$, Nishimura Y. Mechanism of Resistance to Epidermal Growth Factor ReceptorTyrosine Kinase Inhibitors and a Potential Treatment Strategy. Cells 2018;7:212.
14. Li D, Ambrogio L, Shimamura T, et al. BIBW2992, an irreversible EGFR/HER2 inhibitor highly effective in preclinical lung cancer models. Oncogene 2008;27:4702-11.

15. Engelman JA, Zejnullahu K, Gale CM, et al. PF00299804, an irreversible pan-ERBB inhibitor, is effective in lung cancer models with EGFR and ERBB2 mutations that are resistant to gefitinib. Cancer Res 2007;67:11924-32.

16. Wu SG, Liu YN, Tsai MF, et al. The mechanism of acquired resistance to irreversible EGFR tyrosine kinase inhibitor-afatinib in lung adenocarcinoma patients. Oncotarget 2016;7:12404-13.

17. Katakami N, Atagi S, Goto K, et al. LUX-Lung 4: a phase II trial of afatinib in patients with advanced nonsmall-cell lung cancer who progressed during prior treatment with erlotinib, gefitinib, or both. J Clin Oncol 2013;31:3335-41.

18. Skoulidis F, Papadimitrakopoulou VA. Targeting the Gatekeeper: Osimertinib in EGFR T790M MutationPositive Non-Small Cell Lung Cancer. Clin Cancer Res 2017;23:618-22.

19. Cross DAE, Ashton SE, Ghiorghiu S, et al. AZD9291, an Irreversible EGFR TKI, Overcomes T790M-Mediated Resistance to EGFR Inhibitors in Lung Cancer. Cancer Discov 2014;4:1046-61.

20. Ballard P, Yates JW, Yang Z, et al. Preclinical Comparison of Osimertinib with Other EGFR-TKIs in EGFR-Mutant NSCLC Brain Metastases Models, and Early Evidence of Clinical Brain Metastases Activity. Clin Cancer Res 2016;22:5130-40.

21. Mok TS, Wu YL, Ahn MJ, et al. Osimertinib or PlatinumPemetrexed in EGFR T790M-Positive Lung Cancer. N Engl J Med 2017;376:629-40.

22. Nie K, Zhang Z, Zhang C, et al. Osimertinib compared docetaxel-bevacizumab as third-line treatment in EGFR T790M mutated non-small-cell lung cancer. Lung Cancer 2018;121:5-11.

23. Wu YL, Ahn MJ, Garassino MC, et al. CNS Efficacy of Osimertinib in Patients With T790M-Positive Advanced Non-Small-Cell Lung Cancer: Data From a Randomized Phase III Trial (AURA3). J Clin Oncol 2018;36:2702-9.

24. Yang JCH, Ahn MJ, Kim DW, et al. Osimertinib in Pretreated T790M-Positive Advanced Non-SmallCell Lung Cancer: AURA Study Phase II Extension Component. J Clin Oncol 2017;35:1288-96.

25. Goss G, Tsai CM, Shepherd FA, et al. Osimertinib for pretreated EGFR Thr790Met-positive advanced nonsmall-cell lung cancer (AURA2): a multicentre, open-label, 
single-arm, phase 2 study. Lancet Oncol 2016;17:1643-52.

26. Zhou C, Wang M, Cheng Y, et al. P3.02b-096 Osimertinib (AZD9291) in Asia-Pacific Patients with T790M

Mutation-Positive Advanced NSCLC: Open-Label Phase II Study Results. J Thorac Oncol 2017;12:S1250.

27. Julian PTH, Green S. Cochrane Handbook for Systematic Reviews of Interventions Version 5.2.0. UK: John Wiley \& Sons, 2011.

28. Wells GA, Shea B, O'Connell D, et al. The NewcastleOttawa Scale (NOS) for Assessing the Quality of Nonrandomized Studies in Meta-Analysis. (accessed 10 August 2019). Available online: http://www.ohri.ca/ programs/clinical_epidemiology/oxford.asp

29. Yoshida T, Kuroda H, Oya Y, et al. Clinical outcomes of platinum-based chemotherapy according to T790M mutation status in EGFR-positive non-small cell lung cancer patients after initial EGFR-TKI failure. Lung Cancer 2017;109:89-91.

30. Soria JC, Kim SW, Wu YL, et al. Gefitinib/chemotherapy vs chemotherapy in EGFR Clinical Drug Investigation mutation-positive NSCLC after progression on 1st line gefitinib (IMPRESS study): final overall survival (OS) analysis. Ann Oncol 2016;27:vi416.

31. Yamane Y, Shiono A, Ishii Y, et al. Treatments and outcomes of advanced/recurrent non-small cell lung cancer harboring the EGFR T790M mutation: a retrospective observational study of 141 patients in Japan. Jpn J Clin Oncol 2016;46:1135-42.

32. Mann H, Andersohn F, Bodnar C, et al. Adjusted Indirect Comparison Using Propensity Score Matching of Osimertinib to Platinum-Based Doublet Chemotherapy in Patients with EGFRm T790M NSCLC Who Have Progressed after EGFR-TKI. Clin Drug Investig 2018;38:319-31.

33. Takeda M, Okamoto I, Nakagawa K. Pooled safety analysis of EGFR-TKI treatment for EGFR mutation-positive non-small cell lung cancer. Lung Cancer 2015;88:74-79.

34. Goss G, Tsai CM, Shepherd FA, et al. CNS response to osimertinib in patients with T790M-positive advanced NSCLC: pooled data from two phase II trials. Ann Oncol
2018;29:687-93.

35. Hata A, Katakami N, Yoshioka H, et al. Spatiotemporal T790M Heterogeneity in Individual Patients with EGFR-Mutant Non-Small-Cell Lung Cancer after Acquired Resistance to EGFR-TKI. J Thorac Oncol 2015;10:1553-9.

36. Brastianos PK, Carter SL, Santagata S, et al. Genomic Characterization of Brain Metastases Reveals Branched Evolution and Potential Therapeutic Targets. Cancer Discov 2015;5:1164-77.

37. Reungwetwattana T, Nakagawa K, Cho BC, et al. CNS Response to Osimertinib Versus Standard Epidermal Growth Factor Receptor Tyrosine Kinase Inhibitors in Patients With Untreated EGFR-Mutated Advanced Non-Small-Cell Lung Cancer. J Clin Oncol 2018;JCO2018783118.

38. Soria JC, Ohe Y, Vansteenkiste J, et al. Osimertinib in Untreated EGFR-Mutated Advanced Non-Small-Cell Lung Cancer. N Engl J Med 2018;378:113-25.

39. Ercan D, Choi HG, Yun CH, et al. EGFR Mutations and Resistance to Irreversible Pyrimidine-Based EGFR Inhibitors. Clin Cancer Res 2015;21:3913-23.

40. Wang Z, Yang JJ, Huang J, et al. Lung Adenocarcinoma Harboring EGFR T790M and In Trans C797S Responds to Combination Therapy of First- and Third-Generation EGFR TKIs and Shifts Allelic Configuration at Resistance. J Thorac Oncol 2017;12:1723-7.

41. Wang Z, Wu YL. Re-emerging C797S In Trans and Rechallenge of Osimertinib With Erlotinib. J Thorac Oncol 2019;14:e81-2.

42. Arulananda S, Do H, Musafer A, et al. Combination Osimertinib and Gefitinib in C797S and T790M EGFRMutated Non-Small Cell Lung Cancer. J Thorac Oncol 2017;12:1728-32.

43. Rangachari D, To C, Shpilsky JE, et al. EGFR-Mutated Lung Cancers Resistant to Osimertinib through EGFR C797S Respond to First-Generation Reversible EGFR Inhibitors but Eventually Acquire EGFR T790M/C797S in Preclinical Models and Clinical Samples. J Thorac Oncol 2019;14:1995-2002.
Cite this article as: Cui S, Zhang Y, Liu L, Li Y, Zhou R, Huang X, Cao S, Huo X, Wang N. The efficacy and safety of Osimertinib in advanced non-small cell lung cancer patients with Thr790Met resistance mutations: a systematic review and meta-analysis. Ann Palliat Med 2021;10(2):1851-1860. doi: 10.21037/apm-20-1357 


\section{Supplementary}

\section{Appendix 1}

We evaluated the methodological quality of the included articles according to Ottawa scale, with detailed information as follows:

\section{NEWCASTLE - OTTAWA QUALITY ASSESSMENT SCALE}

\section{Selection ( $0-4$ points):}

1) Representativeness of the exposed cohort

a) truly or somewhat representative of the average level in the community(1 point).

b) selected group of users or no description of the derivation of the cohort( 0 point).

2) Selection of the non-exposed cohort

a) drawn from the same community as the exposed cohort(1 point).

b) drawn from a different source or no description of the derivation of the non-exposed cohort $(0$ point $)$.

3) Ascertainment of exposure

a) secure record or structured interview(1 point).

b) written self report or no description(0 point).

4) Demonstration that outcome of interest was not present at start of study

a) yes (1 point).

b) no (0 point).

\section{Comparability (0-2 points):}

1) Comparability of cohorts on the basis of the design or analysis

a) study controls for the most important factor and any additional factor(1 point).

b) study controls for any additional factor(1 point).

c) study controls without the most important factor or any additional factor(0 point).

\section{Outcome (0-3 points):}

1) Assessment of outcome

a) independent blind assessment or record linkage(1 point).

b) self report or no description(0 point).

2) Was follow-up long enough for outcomes to occur

a) yes (1 point).

b) no (0 point).

3) Adequacy of follow up of cohorts

a) complete follow up or subjects lost to follow up unlikely to introduce bias(1 point).

b) follow up rate $<80 \%$ and no description of those lost, or no statement $(0$ point $)$.

\section{Quality assessment:}

1) The quality of each study was defined as
a) "poor" (total score $<4$ ).
b) "fair" ( $4 \leq$ total score $\leq 6)$.
c) "good" ( $7 \leq$ total score $\leq 9)$. 
Table S1 Quality assessment of eligible single-arm studies using the Newcastle-Ottawa quality assessment scale

\begin{tabular}{lcccc}
\hline Study (year) & Selection & Comparability & Outcome & Total scores \\
\hline Yang 2017 & 3 & 0 & 3 & 6 \\
Goss 2016 & 3 & 0 & 3 & 6 \\
Zhou 2017 & 3 & 0 & 3 & 6 \\
\hline
\end{tabular}

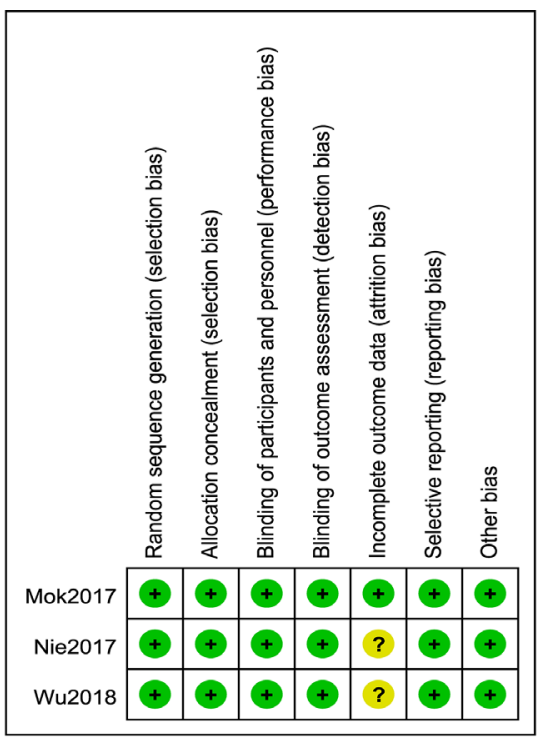

A The risk of bias graph

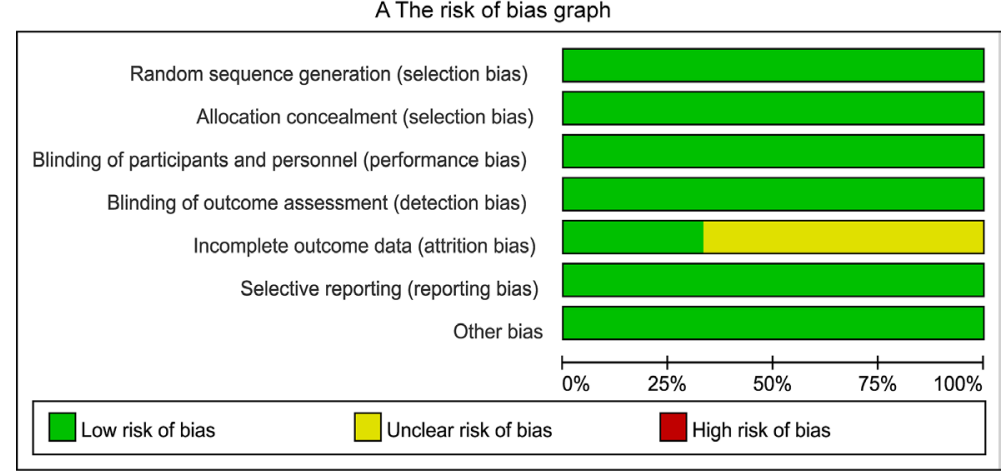

B The risk of bias summary

Figure $\mathbf{S 1}$ The risk of bias in the included RCTs. 